Filigrane

Écoutes psychanalytiques

\title{
Quelle psychanalyse dans ce siècle ? Entretien avec Laurence Kahn
}

\section{Jacques Mauger}

Volume 24, numéro 1, printemps 2015

URI : https://id.erudit.org/iderudit/1033084ar

DOI : https://doi.org/10.7202/1033084ar

Aller au sommaire du numéro

\section{Éditeur(s)}

Revue Santé mentale au Québec

ISSN

1192-1412 (imprimé)

1911-4656 (numérique)

Découvrir la revue

\section{Citer ce document}

Mauger, J. (2015). Quelle psychanalyse dans ce siècle ? Entretien avec Laurence Kahn. Filigrane, 24(1), 73-89. https://doi.org/10.7202/1033084ar 


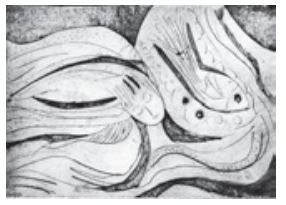

\title{
Quelle psychanalyse dans ce siècle? Entretien avec Laurence Kahn ${ }^{1}$
}

\author{
Jacques Mauger
}

Jacques Mauger: Alors chère Laurence, je te redis tout le plaisir que j'ai à te retrouver à Montréal après toutes ces années. J'apprécie que tu aies accepté d'avoir cet entretien autour de ton dernier livre ${ }^{2}$, à l'occasion de ta présence à ce congrès international d'analystes francophones.

Nous allons aujourd'hui essayer de voir comment ce nouveau livre a aussi des antériorités, une histoire, par laquelle j'aimerais bien que l'on commence notre entretien. Peut-être dire d'abord un mot de ton histoire professionnelle, ta formation, en insistant bien sûr sur ce que je sais être ta formation de base: historienne et helléniste qui a travaillé pendant plus de dix ans avec une équipe extraordinaire dirigée par Jean-Pierre Vernant.

Laurence Kahn: Tout d'abord, merci infiniment de m'avoir conviée à cet entretien. Pour ce qui est de l'antériorité, j'ai effectivement déjà travaillé avec vous, avec toi, en 2003 puis en 2005, lorsque je suis venue à Montréal. Une conférence publique en 2003, puis les entretiens de La Sapinière en 2005. Depuis, le travail en commun n'a jamais cessé: nous avons toujours communiqué et travaillé ensemble. C'est important parce que je ne saurais si c'est le fait d'avoir parlé à l'étranger qui m'a donné l'audace de commencer à dire certaines choses que je pensais concernant le devenir de la psychanalyse contemporaine. Ma vision du devenir de la psychanalyse contemporaine a évidemment tout à voir, comme un après-coup, avec ce qu'a été ma formation d'origine sur laquelle tu m'interroges. Je suis en effet helléniste, ce qui implique des études classiques (allemand, latin, grec) et des études d'histoire. Mon doctorat portait sur la mythologie grecque; sur les conseils de Jean-Pierre Vernant, j'ai travaillé sur l'Hymne homérique à Hermès, travail qui s'intégrait dans la recherche menée par le laboratoire qu'il dirigeait sur les figures de la ruse en Grèce ancienne. Ce fut à la fois une très grande discipline de travail et une expérience qui est restée ancrée dans l'horizon de ce qui m’a aidée à penser la psychanalyse. Nécessairement, quand j'aborde la 
psychanalyse, le travail en détail par exemple sur la traduction d'CEdipe roi, ou bien le travail avec Nicole Loraux — une grande amie que j'ai côtoyée pendant des dizaines d'années - fonctionnent comme une espèce d'arrière-plan très profond.

JM: Il est important bien sûr de souligner ta formation de base, car ce fut tellement déterminant dans ce que tu as écrit, surtout à partir de 2003, à propos de cette question du tragique en voie de disparition. La question se pose par rapport à la pratique théorique et clinique de la psychanalyse... Cela t'habitait avant même de commencer ta formation en psychologie; ensuite tu as travaillé avec les enfants et tu es devenue analyste... Tu étais donc très immergée dans la pratique, mais constamment sollicitée par cette question du tragique chez les écrivains de la Grèce antique.

LK: Il y a beaucoup de choses dans ce que tu dis. À la fois, il y a un mouvement de rupture et un mouvement de retrouvailles. Le mouvement de rupture est pour moi extrêmement net: il s'est matérialisé lorsque j'ai quitté le laboratoire de Jean-Pierre Vernant; j'ai donné ma démission pour devenir analyste, pour pratiquer la psychanalyse, pour assurer une consultation, pour être immergée dans la chose analytique. Il me semblait, surtout avec le poids de Vernant qui était vraiment un homme de la rationalité, que jamais je ne parviendrais à faire ce métier que je désirais très ardemment pratiquer, si je restais dans ce mode de pensée logique et rationnel. D’autant que Vernant pensait la naissance de la pensée grecque, la naissance de la démocratie et la manière dont la tragédie correspondait à l'élaboration du statut de citoyen face à la question de la décision dans les termes d'une histoire des mentalités. Il n'envisageait l'irrationalité de la folie humaine, c'est-à-dire l'hubris, que sous l'aspect des solutions qui y étaient apportées. On se demandait alors peu ce qui, dans la notion même d'hubris, pouvait se rapporter à l'action de l'inconscient. Donc, j'ai quitté le laboratoire tout en continuant à travailler avec eux, et surtout tout en continuant à travailler avec Nicole Loraux.

$\mathrm{JM}$ : Mais à ce moment-là, tu avais déjà commencé une lecture approfondie de l'œuvre de Freud?

LK: Complètement! Même dans Hermès passe! En fait, mes ennuis — internes et externes - ont commencé assez tôt, ne serait-ce que parce que, pendant que je faisais mes études d'histoire et que je lisais les mythes grecs, je lisais parallèlement Freud. Si bien que, dans Hermès passe, j'avais déjà commencé à développer une hypothèse à propos de l'action du mythe - hypothèse qui de par sa source freudienne, n'enchantait absolument pas Vernant, Vidal-Naquet et Detienne. Cela concernait la forme. Je m'étais 
rendu compte que le texte de l'Hymne homérique à Hermès procédait par endroit à une inversion complète de l'emploi du vocabulaire normalement utilisé pour le sacrifice, avec la distinction des deux univers: le sacré du côté des Dieux et le sacré du côté des hommes. C'était extrêmement important à mes yeux parce que, d'une part, un bon nombre d'hellénistes s'étaient servis du sacrifice d'Hermès comme d'un véritable sacrifice, sans s'apercevoir que c'était en même temps un anti-sacrifice. Et il était aussi très intéressant de constater qu'Hermès fait ce qu'il dit qu'il veut faire, et ce à quoi il servira comme Dieu dans la mythologie. Il y avait là une pratique de l'acte, qui prenait corps dans la pratique des mots, c'est-à-dire dans l'ambigüité des mots. Tout cela s'appuyait - le plus discrètement que je le pouvais, quoique très nettement - sur Freud que j'avais évidemment en tête à cette époque, et dont je m'étais inspirée presque tout de suite. Puis, j'ai recommencé une deuxième fois à propos des sirènes, lors d'une intervention dans un congrès en 1977 (j'avais alors repris mes études de psychologie et probablement déjà soutenu ma thèse). J'y ai présenté quelque chose, là aussi, à l'encontre de la grande tradition des hellénistes de l'École dite de Paris. J'ai déplié tout le champ sémantique du son, de la voix, de la séduction, du charme, en référence à l'œuvre de Kafka, Le silence des sirènes. J'avais repris l'analyse des jeunes filles que sont les sirènes, c'est-à-dire des Parthénoi, en tant que figures de l'effroi et de la séduction; de nouveau, on trouvait que j'exagérais un petit peu avec la psychanalyse. Et je me suis dit que j'allais continuer à exagérer beaucoup avec la psychanalyse!

$\mathrm{JM}$ : Donc, c'est là la rupture?

LK: C'est là la rupture, absolument!

JM: Parce qu'Hermès, c'était ton premier livre, n'est-ce pas?

LK: Hermès était mon premier livre. J'avais terminé de l'écrire en 1974 et il a été publié en 1978; puis j'ai quitté les Hautes Études en 1979.

JM: Après ça, il s'est passé quand même tout un temps...

LK: ... où j'ai appris mon métier d'analyste.

JM: Oui, justement. J'aimerais que nous nous attardions à ton rapport du Congrès des psychanalystes de langue française (CPLF) tenu à Paris, en 2001: L'action de la forme. J'aimerais en parler parce que d'une part, tu l'as retravaillé et publié l'an dernier sous le titre de L'écoute du psychanalyste. Par ailleurs, tu me disais justement comment ce rapport avait été déterminant, en tant qu'occasion de reprendre une lecture extrêmement rigoureuse de Freud...

LK: Oui, déterminant. C'est assez intéressant que tu enchaînes sur L'action de la forme, puisque nous venons de parler du moment où j'avais commencé 
à aborder un usage du travail de Freud sur la forme, la déformation et une capacité d'effectivité de la forme elle-même, et ce, avec le mythe. Quand je montrais que le mythe était en train d'effectuer dans l'usage même des mots ce qu'il disait en vue de la structuration d'une cosmogonie, j'élaborais déjà cette notion de l'action d'une forme et de l'action des mots.

Par ailleurs, j'ai appris mon métier entre 1980-1990: j'ai dû devenir membre de l'Association psychanalytique de France vers 1992. À cette époque, j'ai beaucoup travaillé avec J-B. Pontalis et ce, pendant des années. Ce travail a été entièrement tapissé par la lecture de Freud, avec chaque fois un véritable étonnement à redécouvrir l'extrême richesse de ces textes. Parfois, je me dis que Freud, c'est comme Aristote. C'est très simple: on va essayer de dire qu'il est trop vieux, qu'il est déjà usé, qu'il est théoriquement très fatigué, qu'on n'a plus besoin de tout cet appareil, etc. Mais dans un millénaire, on s'apercevra que les embryons de ce qu'on perçoit comme des découvertes sont déjà dans ces textes d'une grande inventivité, qui mettent en place de nouvelles structures de pensée. Donc, oui, une lecture assidue de Freud...

Dans L'écoute de l'analyste, toute la fin de mon rapport de 2001 a été complètement retravaillée: j'y ai étoffé la question de l'écoute proprement dite, c'est-à-dire ce que l'analyste mobilise quand il écoute son patient. De fait, il ne se contente pas de traduire les contenus sémantiques des énoncés avec un système de renvoi terme à terme. Il ne s'agit pas d'une écoute immédiatement traductive de ce qui est dit par le patient, ni non plus d'une espèce d'émerveillement devant les affects du patient avec la croyance que le noyau affectif de ce qui est dit contiendrait le noyau de vérité. L'écoute de l'analyste a été pour moi une étape absolument essentielle. Je crois que je n'aurais jamais pu écrire Le psychanalyste apathique et le patient postmoderne si je n'avais pas fait cet énorme travail en amont, et si je ne m'étais pas moi-même exposée dans la compréhension de Freud et de l'usage que l'on peut en faire, en discutant avec un certain nombre d'interlocuteurs. Par exemple, dans L'écoute de l'analyste, je discute longuement certaines positions de Laplanche et de Widlöcher. Donc une discussion avec mes maîtres et mes collègues...

En fait, tout ce travail se chevauche: le rapport au CPLF en 2000-2001, les premiers fichiers pour Le psychanalyste apathique en 2006, le commencement du travail en vue de la publication de L'écoute de l'analyste vers 2007, finalement paru en $2012 \ldots$

JM: Et puis, il faut rappeler ce qu'on disait tout à l'heure: en 2003, dans les années qui ont suivi immédiatement L'action de la forme, tu es venue à 
Montréal, invitée par la Société psychanalytique de Montréal, et tu as donné cette conférence intitulée Destin de destin qui, par bien des aspects, était déjà annonciatrice de ce que tu as développé par la suite dans le livre qui vient de paraître. Ce livre, ce fut pour moi, d'abord et avant tout, une conférence que j'ai eu la chance de lire et de travailler de près, parce qu'on m'avait demandé d'en faire la discussion. Je t'ai alors rencontrée, d'une certaine façon, à travers ce que tu écrivais. Avant de parler de ton plus récent livre, reprenons certains aspects déjà introduits lors de la conférence de Montréal dans Destin de destin. D’emblée était posée la question de ce que Freud avait vécu d'espérance et de désespérance à son époque. D'abord, l'espérance de cet homme des Lumières qui croyait à une certaine forme de progrès par la culture. Puis la désespérance, à travers l'expérience de la guerre: ses enfants qui sont allés au front, certains amis qui sont devenus des ennemis. Il découvre une tragédie à l'intérieur, à la fois de lui-même et des gens dont il est très proche, que l'on retrouve dans son œuvre, dès les années 1914-1915 et dans l'après-guerre. J'aimerais que tu rappelles ce que tu avais déjà introduit à ce propos, en 2003, quand tu étais venue nous rencontrer ici à Montréal.

LK: Quand tu dis que Freud est un homme qui a vécu dans son temps, il est vrai qu'il a vécu pleinement ce moment de bascule. Ils ont d'ailleurs été nombreux à vivre ce moment de bascule absolument terrible: Valéry, Husserl, Freud, Ortega y Gasset, Thomas Mann... De grands penseurs qui, d'un seul coup, se sont retrouvés face à cette déchirure radicale du monde. Quand on prend par exemple l'adresse de Freud au congrès de Nuremberg en 1910, c'est vraiment le discours d'un Aufklärer, c'est-à-dire la découverte de quelque chose concernant l'humain qui modifie radicalement la vision de l'homme. Outre la méthode thérapeutique, il y a une dimension anthropologique dans la découverte freudienne, dès l'origine avec la lecture d'Oedipe et de Hamlet. Très vite Freud a l'intuition que la découverte va aller au-delà des patients névrosés, et pourra être mise au service des masses.

D'ailleurs, il est intéressant de constater que l'Institut de formation de Berlin, qui est à peu près fondé à cette période-là et dont j'ai analysé de près la structure, propose des consultations publiques, des formations et des services offerts par les jeunes cliniciens qui prennent leurs patients en analyse gratuitement avec un système de supervision. C'est un bijou d'intelligence, d'ouverture au monde, de responsabilité, et tout cela en référence au modèle freudien de compréhension de l'appareil psychique. Autrement dit, à cette époque, il n'y a absolument aucun écart entre ce que Freud est en train de découvrir et ce que ses disciples travaillent avec lui (parce que ce sont des 
disciples très actifs) au plan d'une anthropologie globale, d'une part, et ce qu'ils mettent en ouvre pratiquement, dans les cures, d'autre part. C'est extrêmement important puisqu'au fond, cela s'oppose à l'idée que ce que Freud a élaboré est beaucoup trop compliqué pour s'en servir partout, tout le temps... Selon moi, ce n'est pas trop lourd du tout! Dans la polyclinique de Berlin, ils ont entrepris des prises en charge d'enfants, d'adultes, en face à face, allongés, etc., avec la théorie freudienne. Pourquoi y renoncerait-on? Ça, c'est le Freud Aufklärer.

$1914 \ldots$ c'est l'effondrement de l'Association internationale de psychanalyse. Les Hollandais, les Anglais, les Allemands, les Autrichiens s'aperçoivent qu'ils étaient convaincus (comme les socialistes l'étaient) que leurs idéaux étaient une évidence commune pour tous et qu'il n'y avait absolument pas de problème: ils avaient l'éternité devant eux. À ceci près que, du jour au lendemain, Anna Freud qui était en stage en Angleterre, à l'été 1914, est obligée de rentrer, bride abattue, à Vienne. Jones reste en contact le temps qu'il peut puis, ensuite, impossible: les Anglais n'ont plus le droit de communiquer avec les Allemands et les Autrichiens.

JM: C'est une révélation?

LK: C'est une véritable révélation. Deuxième aspect de la guerre de 1914: puisque tous les fils de Freud sont au front, celui-ci — qui est sans pitié avec lui-même - se dit: quelle folie de m'apercevoir que je voudrais la victoire! Cela ouvre en soi un nouveau champ de réflexion. Premièrement, il y a une guerre insensée quant au niveau de meurtres organisés désormais atteints. Deuxièmement, il y a comme la réémergence d'une joie toute-puissante dans ce plan meurtrier. C'est-à-dire que les idéaux, cette fois reliés à l'héroïsme des combattants, ont complètement changé de place et de visage - ce qui à mon avis est l'un des plus importants constats faits par Freud dans les Considérations actuelles sur la guerre et sur la mort.

JM: Son livre de 1915.

LK: Exactement. Avec cette idée que finalement il est moins coûteux psychiquement de céder à la guerre que d'y résister; c'est-à-dire qu'il y a une régulation de l'appareil psychique, et qu'on peut comprendre pourquoi on cède à la guerre même si elle signifie la destruction.

JM: J'ajouterais qu'en 1912, avec Totem et tabou, Freud avait déjà présenté le rapport père-fils, dans le double sens du meurtre et de la nostalgie. Il l'avait introduit au moment de sa rupture avec Jung, le fils répudié. Et ce, juste avant ce que tu viens de rappeler: 1914, cette catastrophe et tout ce qui sera révélé par la guerre qui commence. 
LK: Tu fais bien de me rappeler cet aspect qui est absolument essentiel. Dans les Considérations actuelles sur la guerre et sur la mort, Freud interprète le fait que l'ancêtre meurtrier est absolument intact en nous. On l'a habillé, on l'a fait propre en apparence. Il s'est créé des lois, des États, des États-Nations. Enfin, il y a eu un effort culturel colossal tendant à refouler la dimension du meurtre; et effectivement dans Totem et tabou, il y avait bien l'idée que le pacte social entre les frères après le meurtre du père était un pacte qui organisait l'interdit autour de cette figure paternelle intériorisée - ce qui va devenir l'instance surmoïque — et par suite le refoulement. Donc, quand Freud doit penser le retour du meurtre à grande échelle en 1914-1918, il avance que le meurtrier, notre ancêtre primitif, est absolument intact, vivant en chacun de nous.

JM: Parce que d'une certaine façon, autant il avait découvert toute la question de son désir incestueux vis-à-vis sa fille Mathilde à travers ses rêves au tout début de son œuvre, autant il découvrait, pour ainsi dire, ce qui en était du désir meurtrier du père envers ses fils; le père qui envoie ses fils à la guerre.

LK: D'une certaine façon, oui. Freud est totalement effrayé, d'autant plus lorsqu'un de ses fils est blessé et qu'ils n'ont pas de nouvelle de lui, alors qu'il est sur le front italien qui est un front extrêmement dur au moment où l'Italie entre dans ce grand assaut contre les empires centraux. C'est un moment où il est convaincu que ce fils est mort. Dans les correspondances de Freud, on remarque d'ailleurs la basse continue de l'effroi parce qu'effectivement, ce sont les pères qui ont envoyé les fils à la guerre. Comment ont-ils pu faire cette chose-là? Cela, il le rencontre en lui. Mais Freud étant un homme de courage et de lucidité, il en tire la conclusion que nous ne sommes jamais montés si haut dans nos idéaux et dans notre être civilisé, et qu'en fait nous avons toujours vécu au-dessus de nos moyens. C'est une phrase extraordinaire: nous avons vécu au-dessus de nos moyens. Nous croyions à un état de stabilité de l'éthique, y compris de l'éthique internationale, mais en réalité, nous étions en train de fabriquer de la dette; puis la dette devient la boucherie actuelle. Ce constat est tout à fait remarquable. En vérité, peut-être que je ne me l'étais jamais formulé aussi clairement que tu le fais; mais le contact est absolument direct concernant le père meurtrier. Tout le problème reste de savoir ce qu'il advient des fils meurtriers du père et comment va se penser la question du meurtre après cela.

JM: Et la question de la nostalgie qui est implicite... Tu étais sur le point d'en venir à des textes de l'après-guerre. Il n'est pas sans importance de 
revenir sur le texte de 1919, Un enfant est battu, car c'est bien de cela dont on parle, mais sous l'angle du masochisme.

LK: C'est ça, il faut l'avoir en tête parce que, d'une certaine manière, s'il y a bien un exemple qu'on puisse donner de ce qui a pratiquement disparu du mode de pensée analytique actuel, c'est bien le masochisme, les bienfaits du masochisme. Mais pour penser les bienfaits du masochisme, il faut considérer l'essentiel de la théorie freudienne, qui va de l'excitation à la liaison de l'excitation, sous cette forme de la jouissance douloureuse. À l'heure actuelle, tout cela a été jeté par-dessus bord pour une bonne partie du monde psychanalytique.

Ce qui m'intéresse au fond c'est l'enchaînement de la théorie freudienne après 1914 : l'ancêtre meurtrier en 1915, la névrose de guerre en 1919, puis en 1920 la dimension de la répétition et $A u$-delà du principe de plaisir qui met en place ni plus ni moins que la pulsion de mort. Peu après vont se déplier l'ensemble des éléments de la seconde topique qui intègre, prend, transforme la première topique mais ne la supprime pas (celle-ci demeure un outil de travail important). Tout ce parcours se fait à la fois très rapidement et très lentement, avec l'inflexion vers les textes anthropologiques, lesquels deviennent de plus en plus nombreux chez Freud, mais ce sont tous des textes cliniques: L'avenir d'une illusion, Malaise dans la culture... Et puis on arrive à Moïse et le monothéisme. Tu parlais de Destin de destin... pour moi, l'écart est là : comment passer du caractère intact de l'ancêtre meurtrier à l'idée de barbarie? Quand tu disais au début de l'entretien que Freud est un homme de son temps: oui, il a été un Aufklärer, mais il a connu l'effraction de la désillusion avec la guerre de 1914-1918 et il ne faut pas sous-estimer l'abominable traitement des empires centraux. Ce que je trouve étonnant, quand on lit la correspondance avec Jones, c'est de découvrir ce que contenait le traité de Versailles et qui a compté énormément par la suite: des pays enfermés, où la population n'a pas de passeport, pas le droit de sortir; il faut des visas spéciaux pour aller voir Freud. Donc, sous la forme de la punition, on est à la limite des sans-droit, et puis, le retournement avec la haine dans l'entre-deux-guerres... Alors, qu'est-ce que cela donne dans la psychanalyse de l'après-guerre? C'est tout le problème...

JM: Mais quand on vit au Québec et qu'on lit Freud — du moins, c'est mon expérience -, il est très facile d'oublier l'histoire, le contexte historique immédiat dans lequel son œuvre a été construite. Ça ne tient pas qu’à ça mais on vient de dire que la question de la guerre a été une révélation pour lui et qu'il a pu, à partir de ce qu'il pouvait avoir comme espérance, plonger 
dans ce que des gens ont appelé son pessimisme. Au fond, c'est bien mal nommer la désespérance qui était liée à ce qu'il venait de vivre, et qui allait fonder dans son œuvre théorique des énoncés que sans doute il n'aurait pas pu écrire s'il n'avait pas connu cette époque. Bien sûr, il était très au fait de ce qui s'était passé dans toute la tradition grecque et juive, mais ce fut déterminant pour lui, cette expérience très personnelle dont on vient de rappeler un certain nombre d'événements.

LK: À la fois il faut bien penser que c'est une révélation de l'Histoire et qu'en même temps, le meurtre sous cette forme-là appartient à son histoire personnelle. N'oublions pas que Freud est né en Galicie d'un père juif que les bourgeois viennois pouvaient faire descendre du trottoir en jetant son chapeau d'un revers de main. Avant l'immigration vers Vienne, il a été élevé dans un pays qui fut traversé par les pogroms, ce qui reste dans les mémoires des familles. Donc les questions du meurtre, de l'étranger, du refus de l'altérité appartiennent profondément à son histoire. Toutefois, Freud était un très grand optimiste. Il semble dire que, tant qu'il y aura de la raison pour penser, il y aura une lumière allumée. Pour lui c’était une évidence! Son optimisme, au fond, est celui de l'enfant dont le père est un Juif qui immigre vers Vienne, mais qui reçoit une éducation classique, qui va au Gymnasium, qui fait six heures de grec et cinq heures de latin par semaine. Il faut voir ce qu'était la formation en études classiques à l'époque, en Autriche: c'était tout à fait prodigieux. On est dans un empire, certes; ce n'est pas une démocratie, mais cette ouverture à la culture lui donne un espoir absolument colossal. Donc un double vecteur: il appartient à ce mouvement d'émancipation des masses dans l'Europe de l'époque, mais, dans son histoire, était déjà inscrit le destin de la destruction. «La civilisation vit au-dessus de ses moyens » : il croyait qu'on en avait fini avec ce destin.

JM: Tu as évoqué Moïse et le monothéisme, la fin de l'œuvre freudienne, là où il parle de barbarie, c'est-à-dire ce pacte entre la culture, la civilisation et la barbarie.

LK: C'est là que commence pour moi Le psychanalyste apathique et le patient postmoderne, c'est-à-dire au moment où je me demande de quelle manière Freud aurait pensé la question de la barbarie dans l'après Shoah, à partir de 1945. Comment aurait-il dialogué avec Adorno et Horkheimer par exemple?

Horkheimer avait fait une analyse (il ne faut pas oublier que les locaux de l'École de Francfort ont hébergé l'Institut de psychanalyse de Francfort), et Adorno avait très bien lu Freud. Avant la guerre, en 1928, Adorno fit une première thèse qu'il ne soutiendra pas, et dont le point d'appui était les 
Leçons d'introduction à la psychanalyse de 1917. Et dans de très nombreux textes, disons à partir des années 1955, il va revenir régulièrement à Freud; c'est le cas de Qu'est-ce qu'être Allemand?,Éduquer après Auschwitz, des textes qui ont été publiés dans Modèles critiques. Il se sert alors très régulièrement de Malaise dans la culture, qui devient son texte de référence pour la suite.

En fait, Adorno, lisant Beckett dans l'après-guerre, a déplié le plus possible sa réflexion sur la manière d'élaborer ce qu'a été la Shoah dans la destruction des mots: comment se débrouille-t-on encore avec les mots, une fois que tous les mots ont été détruits? Ceci correspond à l'orientation de l'esthétique adornienne comme lieu de la résistance politique. La lecture de la seconde topique freudienne tapisse par exemple L'innommable. Bref, il suffit de lire les Notes sur Beckett pour retrouver Freud.

JM: Comme tu l'écris dans ton livre, en 1946, Adorno vivait aux États-Unis, et il avait déjà commencé à parler de la psychothérapie. Il confondait psychothérapie et psychanalyse, mais il y voyait déjà une sorte d'accommodation ou de simplification, en faisant ressortir ce que devenait la culture de masse, la massification (c'était son mot) du langage. Cela rejoint ce dont tu viens de parler, ce qu'il a élaboré par la suite. C'est d'ailleurs ce qui introduit ton propos dans ton plus récent livre.

LK: Oui, parce qu'en 1946, Adorno est un immigré. Il part d'abord à New York et il rejoint ensuite à Pacific Palisades une bonne partie de la colonie allemande émigrée sur la côte Ouest (dont Thomas Mann, Schönberg, Horkheimer etc.). Il est alors confronté au devenir de la psychanalyse aux États-Unis et, d'un seul coup, ça n'a absolument plus rien à voir avec ce qui l'a passionnément intéressé dans Freud. Dès lors, il mène une critique au vitriol de la psychanalyse "new look», participant désormais de cette culture du divertissement: la psychanalyse comme cosmétique, comme manière d'adhérer à l'american way of life...

JM: Il critique le culturalisme...

LK: C'est ça... et une sociologisation de la psychanalyse. C'est-à-dire quand le système d'interprétation consiste à prendre en compte l'environnement socioculturel, ce que nous appelons aujourd'hui, dans les nouvelles voies de la psychanalyse la "contextualisation». À l'époque, Adorno repère que ce système, cette intégration de la psychanalyse dans les parties rapidement consommables de la civilisation, va conduire inévitablement à une simplification des modèles, à commencer par cette sociologisation. Si le culturalisme était à l'époque le fer de lance de ce mouvement, on s'aperçoit qu'à l'heure actuelle les mêmes ingrédients se retrouvent sous la forme de la 
contextualisation. Ceci est également vrai de l'extension actuelle de la notion de trauma par laquelle la neurotica reprend du service. Il y a aujourd'hui une vision traumatique de l'histoire de chacun. Effectivement on a tous des ennuis dans la vie, de l'enfance jusqu'à la mort — c'est ce qu'on appelle la vie, d'ailleurs. Mais cette montée en épingle du trauma est telle que la part psychique interne de perlaboration du trauma disparaît complètement au profit de la contextualisation des éléments traumatiques.

JM: L'adaptation de la méthode à travers ce qu'on a appelé très vite, après la guerre, les «nouvelles cliniques», était aussi incluse dans la critique qu'il formulait à ce moment-là.

LK: Ça, je dirais que c'est très important. L'histoire de la nouvelle clinique n'apparaît pas immédiatement. L'adaptation de la méthode va avec cette idée qui s'est installée petit à petit (mais le problème, c'est comment!) que nous aurions affaire à de nouveaux types de patients, ceci étant lié au changement d'univers technologique, social. Puis cette «mythologie» (et je pèse mes mots, mais il me semble que c'est bien une mythologie) a remonté dans le temps, c'est-à-dire que cette supposée transformation était initialement associée aux années 1970, pour finalement être située dans l'immédiat après-guerre. Depuis la seconde guerre mondiale, il y aurait de plus en plus de borderline. Nos patients auraient d'ailleurs complètement changé, et nous serions des psychanalystes extrêmement courageux puisque nous osons prendre en analyse des gens qu'avant la guerre, aucun analyste n'aurait acceptés. Ce faisant, nous aurions besoin de nouveaux outils adaptés à de tels patients dont l'identité est chancelante... Il suffit de lire très soigneusement les cas d'analyse de Freud; est-ce que l'identité de l'homme aux rats, de l'homme aux loups et de Dora — qui ignore qui elle est et ce qu'elle désire comme toute hystérique qui se respecte — n'étaient pas des identités chancelantes? On s'aperçoit qu'à partir du moment où l'on change la voie d'entrée dans le champ analytique ou psychothérapique, parce que ceci est vrai tout autant des psychothérapies, on obtient une vision des cas complètement différente. Ce qui s'est passé dans l'adaptation de la méthode tient à la convergence de plusieurs éléments.

Pour le premier élément, je suis obligée de repasser par l'histoire, afin d'éclairer mon propos. Historiquement, c'est assez intéressant de voir comment naît une théorisation nouvelle de l'appareil psychique et comment un retour en force de la théorie du trauma est apparu à la suite de l'aprèsguerre, lorsque des gens comme Loewenstein (avec son livre sur l'antisémitisme) ou bien Fenichel ou Simmel (je pense au congrès de 1944 à San 
Francisco sur l'antisémitisme) commencent à penser ce qui s'est passé pendant la Shoah; ensuite, silence... Puis en 1964, un texte de Niederland très important, où cet analyste juif émigré aux États Unis essaye de définir ce qu'est un préjudice, plus particulièrement le préjudice non immédiatement visible d'un survivant. À cette époque, les réparations de guerre versées par les Allemands constituent une véritable donnée de réflexion: qu'est-ce qu'être un survivant de la Shoah, et de quelle manière peut-on faire valoir le tort subi même si ce survivant n'a pas perdu bras et jambes? Il va bien falloir réussir à le penser... Et c'est conjointement que la théorie du trauma fait retour dans le champ analytique. L'ensemble tourne autour du fait qu'on (notamment les psychanalystes) s'aperçoit qu'il y a encore de «vrais» survivants, qui ont été dans les camps ou qui ont été des enfants cachés, et qu'il faut les interroger avant qu'ils ne meurent. À ce moment, vers 1970-1972, de grandes enquêtes sont déclenchées (l'enquête Kestemberg, l'enquête Krystal, le groupe Ostow, etc.), lesquelles réunissent des interviews dont les extraits sont ensuite catégorisés.

Ce qui est extraordinaire, c'est que, depuis Niederland jusqu'aux divers comptes rendus des résultats des enquêtes, on voit le modèle du trauma prendre de l'ampleur. Parallèlement, on observe une révision profonde du modèle de fonctionnement de l'appareil psychique puisqu'on en vient à imaginer un appareil psychique si traumatisé qu'il ne lui reste rien pour fonctionner: ça, c'est le «trauma extrême». On se retrouve finalement dans une forme de pathétisation des effets de la Shoah - mouvement qui se prolonge notamment avec le modèle de l'empathie.

Ce mouvement permet par exemple à des gens d'écrire à propos du film Shoah de Lanzmann, que celui-ci a échappé aux camps et que, pénétrant au cœur du «cercle vide» de l'atrocité grâce aux entretiens, il parvient à faire échec à la béance mémorielle engendrée par la dissolution du self que provoque l'expérience traumatique. Le cinéaste composerait avec des fantasmes infantiles qui ont valeur de bouclier protecteur contre l'horrifiante réalité des faits. Le film traduirait donc l'impossibilité d'avoir accès à un processus associatif élaboré, tel que le requièrent les «authentiques souvenirs» et les «authentiques récits», etc. Mais qu'est-ce que ça signifie? Alors qu'il y a précisément une intelligence du montage pour éviter justement le colmatage pur et simple par l'image - qui ferait du film un objet de consommation et qu'il impose la contrainte à passer par le langage, seul outil du témoin. Ces témoins parlent. Ils ne veulent pas qu'on soit dans l'empathie. Ils ont quelque chose à élaborer, sans le spectaculaire, sans l'image et son pathos. 
Or, pour ces auteurs, en l'occurrence Nanette Auerhahn et Dori Laub qui ont écrit toute une série de textes sur le trauma extrême et l'empathie, la situation traumatique extrême à laquelle confronte l'événement historique des camps de concentration exige que l'on abandonne toute référence théorique à l'après-coup. C'est en termes de "fragmentation» psychique, en termes de destruction des liens internes sous le coup de la destruction des liens externes, que doivent être cliniquement abordés les effets d'une telle expérience.

Le trauma, l'empathie, un usage simplifié du langage, une consommation de plus en plus facile de la métapsychologie puisqu'avec le trauma, on a un référent psychique simple - comme s'il était suffisant de ne conserver que le trauma, l'identité et le self qui dès lors est considéré comme l'équivalent de l'identité. Pourtant, en lisant Les embarras de l'identité de Vincent Descombes, on mesure la complexité de ces concepts: la question identitaire est une question extrêmement compliquée, notamment du fait de la prise en compte de la notion de self.

Ce sont tous ces éléments (trauma, empathie, identité/self) qui convergent vers cette idée d'une «nouvelle» clinique, avec un dernier élément essentiel pour comprendre l'ampleur de ce qui se passe à l'heure actuelle: la critique de la psychanalyse comme non scientifique.

Ici, il faut vraiment remonter en amont, dans l'histoire, pour s'apercevoir que les convergences finissent par avoir un sens. Ainsi, Kohut, dans un entretien avec un journaliste du New York Time Magazine, expliquait-il que sa fuite hors d'Autriche avait été un élément déterminant dans l'élaboration de la Self psychology, c'est-à-dire des troubles narcissiques et des pathologies borderlines. Puis, alors que Kohut construit son modèle autour des cicatrices incicatrisables du traumatisme, Schafer qui initialement s'inscrivait dans la veine des psychanalystes de l'Ego psychology, modifie sa propre position: au moment où survient l'attaque en règle de Nagel et Danto dans ce fameux symposium de New York en 1958, où ils font la démonstration que la psychanalyse ne souscrit à aucun des prérequis scientifiques. Finalement, Kohut et Schafer, qui viennent de deux horizons complètement différents, vont s'accorder sur le fait qu'il faut abandonner la métapsychologie freudienne (en délaissant le point de vue économique, soit la référence énergétique à un mode de décharge)... avec de facto une nouvelle orientation vers l'herméneutique.

Ceci a ouvert la porte, entre autres dans les rangs de la philosophie analytique, à un «canardage» absolument systématique de Freud, comme on le 
retrouve dans Wittgenstein, lecteur de Freud, de Bouveresse. Mais cela amène aussi à critiquer les ancrages de ce que Freud appelle des analogies, au-delà de ce qu'il envisage du côté de la métaphore et de l'herméneutique. Ce que fera Bouveresse - et plus largement, la philosophie analytique américaine dans ce qu'elle a de plus rigide - , mais en incluant à cette critique toute une frange de ce qu'on appelle la «French Philosophy» (Derrida, Foucault, Lyotard...) qu'il semble détester.

Au fond, il faut attendre des gens qui relisent les philosophes analytiques américains, des psychanalystes tels Lewis Kirshner (Toward a Postmodern Realism for Psychoanalysis, en 1999) qui a notamment relu Putnam ${ }^{3}$ et qui s'est rendu compte que la question du réalisme se posait dans des termes beaucoup plus compliqués. Il a très bien vu d'où partait la terreur exercée par les suites du Cercle de Vienne ${ }^{4}$, c'est-à-dire le positivisme logique pur et dur. Il a aussi très bien situé la confusion qui règne avec Rorty qui introduit le pragmatisme américain. Car c'est là que se situe l'ouverture à un relativisme qui s'appuie sur l'idée, initialement énoncée par Kuhn puis développée par Foucault, que les véritables changements, ce que Kuhn appelle les révolutions scientifiques, naissent au moment où une théorie obtient un consensus de la société scientifique. La psychanalyse a en partie obtenu ce consensus, et puis cela aurait changé. Mais l'on voit bien ici l'impact du système de validation consensuel par la société.

D'un autre côté, il y a cette idée de Foucault (dans Les mots et les choses) que Rorty reprend mais, lui, sous la forme de l'invalidation de tout discours de vérité: périodiquement, un mode d'articulation du savoir, ce qu'il nomme une épistémè, pivote intégralement - ce qu'il montre avec la notion de travail. Mais Foucault montre bien comment cette notion ne concerne pas un seul champ de pensée. Et de plus, même s'il y a des changements d'épistémè, de configuration épistémique, ça ne veut absolument pas dire que la configuration épistémique antérieure est fausse et hors science. Il montre au contraire, avec l'exemple du système de classification de Cuvier, comment cela va être repris et retravaillé tout autrement; ce qui ne veut nullement dire que ce qui a précédé n'existe plus.

JM: Une des choses que fait ressortir ta lecture de ces philosophes européens, c'est la validation de la position d'abstinence comme étant à la base même de l'écoute analytique.

LK: Il faut bien payer le prix de l'empathie... et du relativisme. Il est là le problème. J'ai presque envie de dire qu'il faut d'abord payer le prix du relativisme puisque, si les énoncés ne sont que des énoncés du moment, si nous 
avons renoncé à la valeur de vérité de l'énoncé, même mise en doute, même mangée de l'intérieur par l'acide du doute — doute qui est tout de même ce que nous vivons jour après jour -, il est pour moi évident que si l'on a renoncé à cela, il ne reste plus que la «gentille» conversation. Je dis gentille parce qu'avec la notion de conversation, la dissymétrie de la situation analytique est du même coup discréditée. L'analyste n'a pas plus de rapport à la vérité que son patient, il n'a pas plus de surplomb que son patient. L'analyste n'a même pas une analyse d'avance - ce que nous nous disons, malgré tout, entre nous - sur son patient. En fait, ce que produit l'analyse est simplement une configuration, la configuration d'un récit sur soi dans l'interaction avec la personne de l'analyste qu'on a — l'interpersonnalisme référant plus encore à la conscience et au sujet que l'intersubjectivisme. Vous changez d'analyste, vous aurez une tout autre configuration. Et comme l'essentiel est centré sur le vécu affectuel, il n'y a plus de levée de l'amnésie. De sorte que le problème de la répétition du passé qui se révélerait éventuellement dans le transfert disparait. Le transfert, c'est un transfert entre patient et analyste au sein duquel toute forme d'autoritarisme de l'analyste a été catégoriquement critiquée.

Le relativisme conduit donc, inévitablement, à une égalité complète des positionnements de l'analyste et du patient, mais à un point qui est parfois renversant. Par exemple, je suis tombée sur des textes de Spezzano expliquant que, lorsqu'il écoute son patient, des idées, des pensées surgissent; et il communique ces idées, ces pensées à son patient en lui disant qu'il espère que celui-ci pourra en faire quelque chose. Où est la responsabilité de l'analyste? En fait, le système relativiste implique intrinsèquement l'adaptation à la demande du patient. Rien ne permet à l'analyste de tenir la position de silence, et ce, même si c'est là le plus difficile dans l'abstinence, laquelle permet d'ouvrir la «coque surmoïque». De fait, c'est par ce biais que l'analyste, apparaissant comme cruel, peut endosser les habits du surmoi (en partie composé des relations d'objet infantiles) et peut de ce fait être attaqué. Ainsi peut se réaliser, s'incarner, la lutte entre le moi et le surmoi, avec en arrièrefond tous les fils qui mènent régressivement au ça. En d'autres termes, la figure paternelle s'impose dans l'analyse par la voie transférentielle de l'attaque contre l'analyste.

JM: À la condition de l'abstinence bien entendu!

LK: À la condition de l'abstinence, car comment voulez-vous attaquer un si gentil analyste qui, lorsqu'on lui demande «Est-ce que vous n'êtes pas malade?» alors qu'il a décommandé une séance, répond: «Ne vous inquiétez pas mais non, je ne suis pas malade!». 
JM: Pour «le bien du patient»...

LK: Il ne faut pas qu'il se fasse du souci inutilement, qu'il connaisse des crises d'angoisse en son absence. Ce pauvre patient est très fragile... Celui-ci pourrait d'ailleurs dire à l'analyste: «Mais alors, votre petit-fils est né? C'est insupportable que vous alliez vous amuser avec vos enfants, vos petitsenfants.» Mais ce n'est pas ce que dit le patient. Ce qu'il dit, c'est: "Vous n'êtes pas malade?» Et on sent le: «et si vous étiez malade?», «si vous étiez bien malade!». Pour atteindre la dimension du meurtre, encore faut-il que l'analyste soit attaquable, c'est-à-dire qu'il permettre l'attaque. Inversement, sur la question de la jouissance, pour atteindre le niveau odipien, encore faut-il que l'analyste soit attaquable sur le silence qu'il fait peser sur ses plaisirs!

En fait, c'est là ce que vise mon ouvrage Le psychanalyste apathique. Être apathique est une qualité bien évidemment, ce n'est pas la nonchalance, ce n'est pas l'insensibilité, c'est même tout le contraire. C'est l'énergie de la froideur - c'est-à-dire l'énergie que génère ce que Dominique Scarfone appelle la passibilité, l'aptitude à être atteint — c'est-à-dire, en fait, l'énergie même de la pensée.

JM: Eh bien, je te remercie encore une fois de ta générosité et surtout, pour ta façon d'incarner tout ce que tu dis. Je me rappelle très bien que j'avais lu L'action de la forme avant de te rencontrer, et après t'avoir entendue parler de ton œuvre en 2003, cela a complètement changé ma lecture de celle-ci. J'ai le sentiment qu'aujourd'hui, il y a quelque chose de cette expérience qu'on a pu relancer, par ta façon de présenter les choses si essentielles, et de toute évidence, si essentielles pour toi.

LK: De même, je veux te remercier, Jacques, de m'avoir offert la possibilité de cet entretien. Que tu prennes comme exemple L'action de la forme, c'est assez drôle parce qu'on ne cesse de me dire que ce livre est horriblement difficile. Ce n'est pas le cas, il a simplement besoin d'être lu avec un petit peu d'attention, et un peu lentement. Bref, je te remercie de m'avoir permis de parler de ces choses. Tu as raison: lorsqu'on explique oralement la façon dont les pensées viennent, la façon dont on bâtit progressivement non pas ces réponses, mais ces questions (puisque finalement, ce ne sont que des questions), cela rend notre pensée différemment accessible.

JM: Justement, je trouve que tu incarnes si bien L'action de la forme. Merci! LK: Merci! 


\section{Notes}

1. Cette entrevue - également disponible en ligne, sur notre site web - a été réalisée le 29 mai 2014 à Montréal, Madame Kahn et Monsieur Mauger participant l'un et l'autre au Congrès des psychanalystes de langue française.

2. Kahn, L. (2014). Le psychanalyste apathique et le patient postmoderne. Paris: Éditions de l'Oliver.

3. Philosophe américain, adepte notamment du réalisme scientifique.

4. Regroupant des tenants de l'empirisme logique. 